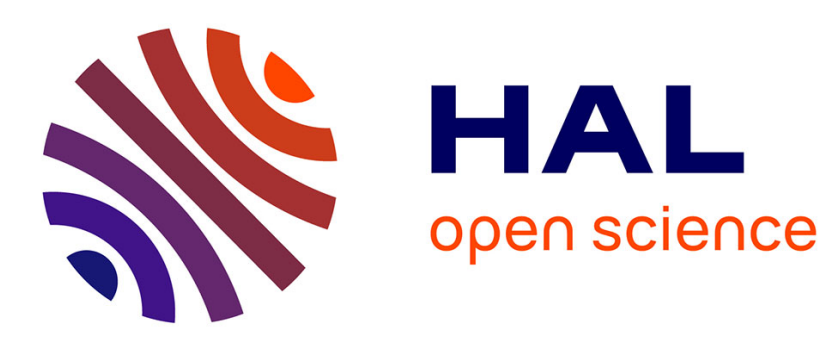

\title{
On the isoplanatic patch size in High Angular Resolution Techniques
}

A. Ziad, E. Aristidi, J. Chabé, J. Borgnino

\section{To cite this version:}

A. Ziad, E. Aristidi, J. Chabé, J. Borgnino. On the isoplanatic patch size in High Angular Resolution Techniques. Monthly Notices of the Royal Astronomical Society, 2019, 10.1093/mnras/stz1541 . hal02283310

\section{HAL Id: hal-02283310 \\ https://hal.science/hal-02283310}

Submitted on 26 Nov 2021

HAL is a multi-disciplinary open access archive for the deposit and dissemination of scientific research documents, whether they are published or not. The documents may come from teaching and research institutions in France or abroad, or from public or private research centers.
L'archive ouverte pluridisciplinaire HAL, est destinée au dépôt et à la diffusion de documents scientifiques de niveau recherche, publiés ou non, émanant des établissements d'enseignement et de recherche français ou étrangers, des laboratoires publics ou privés. 


\title{
On the isoplanatic patch size in high-angular resolution techniques
}

\author{
A. Ziad, ${ }^{1 \star}$ E. Aristidi, ${ }^{1}$ J. Chabé $^{2}$ and J. Borgnino ${ }^{1}$ \\ ${ }^{1}$ Université Côte d'Azur, OCA, CNRS, Lagrange, Parc Valrose, F-06108 Nice Cedex 2, France \\ ${ }^{2}$ Université Côte d'Azur, OCA, CNRS, IRD, Géoazur, 2130 route de l'Observatoire, F-06460 Caussols, France
}

Accepted 2019 May 31. Received 2019 May 22; in original form 2018 November 5

\begin{abstract}
To reach a high performance with an adaptive optics system, we need a calibration using a reference source. This latter should be located in the same isoplanatic domain as the science source. Different techniques and methods have been developed leading to estimations of the isoplanatic patch but all are model-dependent. We developed a new technique for the estimation of the isoplanatic angle based on an extended object. This technique is now part of our new turbulence profile monitor PML (Profiler of Moon Limb) based on the observation of the Moon limb or Sun edge. The first statistics of the isoplanatic angle with this new technique are presented and compared to the exiting techniques based on scintillation measurements or other turbulence parameters such as Fried parameter and/or $C_{n}^{2}$ profile.
\end{abstract}

Key words: Atmospheric effects - site-testing - High Angular Resolution - Interferometers Adaptive Optics.

\section{INTRODUCTION}

The atmospheric turbulence parameters have a strong impact on the image formation through the atmosphere. Indeed, the effect of atmospheric turbulence on wavefronts severely limits the resolution of ground-based astronomical observations. Different high-angular resolution (HAR) techniques have been developed to achieve diffraction-limited resolution of observing instruments, namely adaptive optics (AO) and long baseline interferometry (LBI). Design, performance, and optimization of these HAR techniques require an in-depth knowledge of atmospheric turbulence parameters, notably the seeing (or Fried parameter $r_{0}$ ), the outer scale $\mathcal{L}_{0}$, the coherence time $\tau_{0}$, and the isoplanatic domain $\theta_{0}$. Knowledge of these parameters is also important for future extremely large telescope specifications. Indeed, the Fried parameter and $\mathcal{L}_{0}$ are critical for the deformable mirror in terms of the number of actuators and the required stroke. The knowledge of the wavefront coherence time $\tau_{0}$ is of interest to optimize the HAR technique detector exposure time and the correction frequency of the $\mathrm{AO}$ system. In addition, the choice of the AO reference star must take into account the constraints related to the isoplanatic angle. Indeed, a compromise must be found on the magnitude of the reference star inside the isoplanatic domain. Different techniques have been used for direct and indirect estimation of the isoplanatic field size based on different measured quantities, such as wavefront phase, angle-of-arrival (AA), and scintillation (Fried 1979; Loos \& Hogge 1979; Roddier, Gilli \& Vernin 1982; Ziad et al. 2000). But these different observables do not have straightforward

\footnotetext{
^E-mail: Aziz.ZIAD@unice.fr
}

analytical relationships corresponding to the isoplanatic angle definitions.

In this paper, we present a new direct technique for the estimation of the isoplanatic angle. This new technique is based on the reconstruction of the angular structure function of an extend object such as the Moon and the Sun. The theoretical background of this new technique of isoplanatic angle estimation is detailed in Section 2 and a brief review of the existing methods and techniques using different observable quantities is presented as well. This technique is now part of our new PML profiler (Profiler of Moon Limb; Ziad et al. 2013; Catala et al. 2017) of the turbulence refractive index structure constant $C_{n}^{2}$. The first statistics of the new isoplanatic angle measured with the PML instrument are presented in Section 4. We also provide the first comparisons of the isoplanatic domain measured with different instruments using different techniques based on different observables.

\section{THEORETICAL BACKGROUND}

Estimation of the isoplanatic angle depends strongly on the measured quantity. Indeed, this parameter, as it will be shown in this section, is different when its estimation is based on phase, AA fluctuations, or scintillation. The two first quantities are related by a spatial first derivative $\left[\alpha(x, y)=-\frac{\lambda}{2 \pi} \frac{\partial \varphi(x, y)}{\partial x}, \beta(x, y)=-\frac{\lambda}{2 \pi} \frac{\partial \varphi(x, y)}{\partial y}\right]$ and therefore the measurement direction (angle of the measured AA with the $x$-axis) in the case of AA is crucial. In this section, we want to emphasize the difference of the isoplanatic field deduced from the phase, relevant for interferometry, the one deduced from AA quantities, measured with the Shack-Hartmann of an AO system, and the one obtained from scintillation by means of special filtering. 
Different definitions of the isoplanatic angle $\theta_{0}$ are given in the literature and the most known and used expression is Roddier's one, which is deduced from the spatio-angular correlation of phase complex amplitudes of two point sources separated by an angle $\theta$ (Roddier et al. 1982). Indeed, the isoplanatic angle is obtained when the phase structure function,

$D_{\varphi}(\theta \bar{h})=6.88\left(\frac{\theta \bar{h}}{r_{0}}\right)^{5 / 3}$,

is lower than $1 \mathrm{rad}^{2}$, leading to

$\theta_{0}=0.31 \frac{r_{0}}{\bar{h}}$,

where $r_{0}$ represents the Fried parameter and $\bar{h}$ an equivalent turbulence altitude defined as

$\bar{h}=\left[\frac{\int \mathrm{d} h h^{5 / 3} C_{n}^{2}(h)}{\int \mathrm{d} h C_{n}^{2}(h)}\right]^{3 / 5}$.

This definition is used by the most common profilers, such as Scidar or radio-sounding balloons, to provide estimation of the isoplanatic angle. Indeed, from $C_{n}^{2}$ profile one can estimate the Fried parameter $r_{0}$ by using this equation $\int C_{n}^{2}(h) \mathrm{d} h=0.06 \lambda^{2} r_{0}^{-5 / 3}$ and the equivalent altitude $\bar{h}$ is deduced from equation (3). Then, values of $r_{0}$ and $\bar{h}$ are introduced into equation (2) to provide estimation of the isoplanatic angle $\theta_{0}$.

The error $\Delta \theta_{0}$ on the isoplanatic angle $\theta_{0}$ is obtained by differentiation of equation (2):

$\frac{\Delta \theta_{0}}{\theta_{0}}=\frac{\Delta r_{0}}{r_{0}}+\frac{\Delta \bar{h}}{\bar{h}}$,

where $\Delta r_{0}$ is the error on the Fried parameter estimation and $\Delta \bar{h}$ is the uncertainty on the equivalent altitude. When $\bar{h}$ is obtained from an estimated $C_{n}^{2}$ profile (equation 3 ), this error is given by

$\frac{\Delta \bar{h}}{\bar{h}}=\frac{3}{5}\left[\frac{\sum h_{i}^{5 / 3} \Delta C_{n}^{2}\left(h_{i}\right)}{\sum h_{i}^{5 / 3} C_{n}^{2}\left(h_{i}\right)}+\frac{\sum \Delta C_{n}^{2}\left(h_{i}\right)}{\sum C_{n}^{2}\left(h_{i}\right)}\right] \simeq \frac{6}{5} \frac{\sum \Delta C_{n}^{2}\left(h_{i}\right)}{\sum C_{n}^{2}\left(h_{i}\right)}$,

where $\Delta C_{n}^{2}\left(h_{i}\right)$ is the error on the estimation of $C_{n}^{2}$ at an altitude $h_{i}$. We checked numerically that the two terms between the brackets are almost identical.

On the other hand, the easiest way to measure the isoplanatic angle $\theta_{0}$ is from the scintillation of a single star observed through a pupil of $10 \mathrm{~cm}$ diameter and a central obstruction of $4 \mathrm{~cm}$. The principle of the calculation is based on the similarity of the theoretical expressions of $\theta_{0}$ and the scintillation index (Loos \& Hogge 1979; Ziad et al. 2000). $\theta_{0}$ is obtained in arcsec for a wavelength $\lambda=500 \mathrm{~nm}$ from the following formula:

$\theta_{0}^{-5 / 3}=A(\cos z)^{-8 / 3} s$,

where $A=14.87$ is computed numerically from equations (19) and (21) of Ziad et al. (2000). $z$ is the zenithal distance of the star.

The errors on the $\theta_{0}$ estimation from this scintillation technique have been evaluated and discussed by Ziad et al. (2000) and Aristidi et al. (2019).

In this section, we want to point out the possibility of a direct measurement of the isoplanatic angle from an extended object. Indeed, a direct and easy way to have access to the isoplanatic angle estimation is the use of angular correlation of the measured quantities such as phase, AA, or scintillation. For example, correlation of AA fluctuations obtained on an extended object such as the Moon limb or the Sun edge is very efficient to provide estimation of the isoplanatism range. The correlations of these AA fluctuations for different angular separation $\theta$ along the extended object are well represented in the AA structure function as

$D_{\alpha}(\theta)=2\left[\Gamma_{\alpha}(0)-\Gamma_{\alpha}(\theta)\right]$,

where $\Gamma_{\alpha}(\theta)$ indicates the angular transverse covariance for the AA fluctuations of two sources (or positions on the extended object) separated by an angle $\theta . \Gamma_{\alpha}(\theta)$ is related to the spatial covariance $C_{\alpha}$ as (Ziad et al. 2013)

$\Gamma_{\alpha}(\theta)=\int_{0}^{+\infty} \mathrm{d} h C_{n}^{2}(h) C_{\alpha}(\theta h)$.

In this equation, $C_{\alpha}$ is the normalized spatial covariance, which in the case of the von Kàrmàn model for a baseline $\varrho=\theta h$, a subaperture diameter $D$, and a single layer at altitude $h$ is given by Avila et al. (1997) as

$$
\begin{aligned}
C_{\alpha}(\varrho)= & 1.19 \sec (z) \int \mathrm{d} f f^{3}\left(f^{2}+\frac{1}{\mathcal{L}_{0}(h)^{2}}\right)^{-11 / 6} \\
& {\left[J_{0}(2 \pi f \varrho)-\operatorname{Cos}(2 \gamma) J_{2}(2 \pi f \varrho)\right]\left[2 \frac{J_{1}(\pi D f)}{\pi D f}\right]^{2}, }
\end{aligned}
$$

where $f$ is the modulus of the spatial frequency, $z$ is the zenithal distance, and $\mathcal{L}_{0}(h)$ is the outer scale profile. $\gamma$ is the angle between the direction of the AA fluctuations and the baseline. For transverse AA fluctuations (perpendicular to the baseline), $\gamma=\pi / 2$.

A definition of a wavefront isoplanatic angle $\theta_{\alpha}$ can be derived from the structure function of AA fluctuations $D_{\alpha}(\theta)$, in a similar way than in Ziad et al. (2012) for the AA coherence time. Hence, we define $\theta_{\alpha}$ as the angle for which $D_{\alpha}(\theta)$ is equal to the $\frac{1}{\kappa}$ of its saturation value, with $\kappa$ a constant:

$D_{\alpha}\left(\theta_{\alpha}\right)=\frac{2 \Gamma_{\alpha}(0)}{\kappa}$.

This wavefront isoplanatic angle $\theta_{\alpha}$ is obtained directly and nonmodel-dependent. Using von Kàrmàn model and the same reasoning in Ziad et al. (2012) in the case of a small telescope of diameter $D$, we found

$\theta_{\alpha} \simeq \frac{D}{\bar{h}} \sqrt{\frac{2.62}{\kappa} \frac{\left[1-1.04\left(\frac{\pi D}{\mathcal{L}_{0}}\right)^{1 / 3}\right]}{2+\cos (2 \gamma)}}$ for $\theta_{\alpha}<D / \bar{h}$.

For large AA isoplanatic angle, we have a simplified expression:

$\theta_{\alpha} \simeq \frac{D}{\bar{h}}\left[\frac{7.30 \kappa^{-1}\left(\frac{\pi D}{\mathcal{L}_{0}}\right)^{1 / 3}+7.01\left(1-\kappa^{-1}\right)}{5-\cos (2 \gamma)}\right]^{-3}$ for $\theta_{\alpha}>D / \bar{h}$.

Examples of angular structure functions of AA fluctuations $D_{\alpha}(\theta)$ deduced from the Sun edge are shown in Fig. 1. The saturation level of $D_{\alpha}(\theta)$ is given by AA variance, which is deduced from equation ( 8 ) for $\theta=0$. These structure functions have been deduced from images of two Sun edges obtained with the PML instrument at the Calern Observatory on 2018 June 18. The fold of the saturation by a constant $\kappa=e$ leads to $\theta_{\alpha}=7.7$ arcsec. Including this value of $\theta_{\alpha}$ in equations (11) and (12), we obtained an equivalent altitude around $\bar{h}=2897 \mathrm{~m}$ for an outer scale of $20 \mathrm{~m}$. Using equation (2) and knowing that the seeing was 2.59 arcsec, this equivalent altitude leads to an isoplanatic angle equal to $\theta_{0}=0.92 \operatorname{arcsec}$ at $\lambda=$ $0.5 \mu \mathrm{m}$. However, the value obtained from the profile of $C_{n}^{2}$ using equations (3) and (2) is of $\theta_{0}=1.03$ arcsec. These values are in good agreement, proving the pertinence of the technique presented above. 


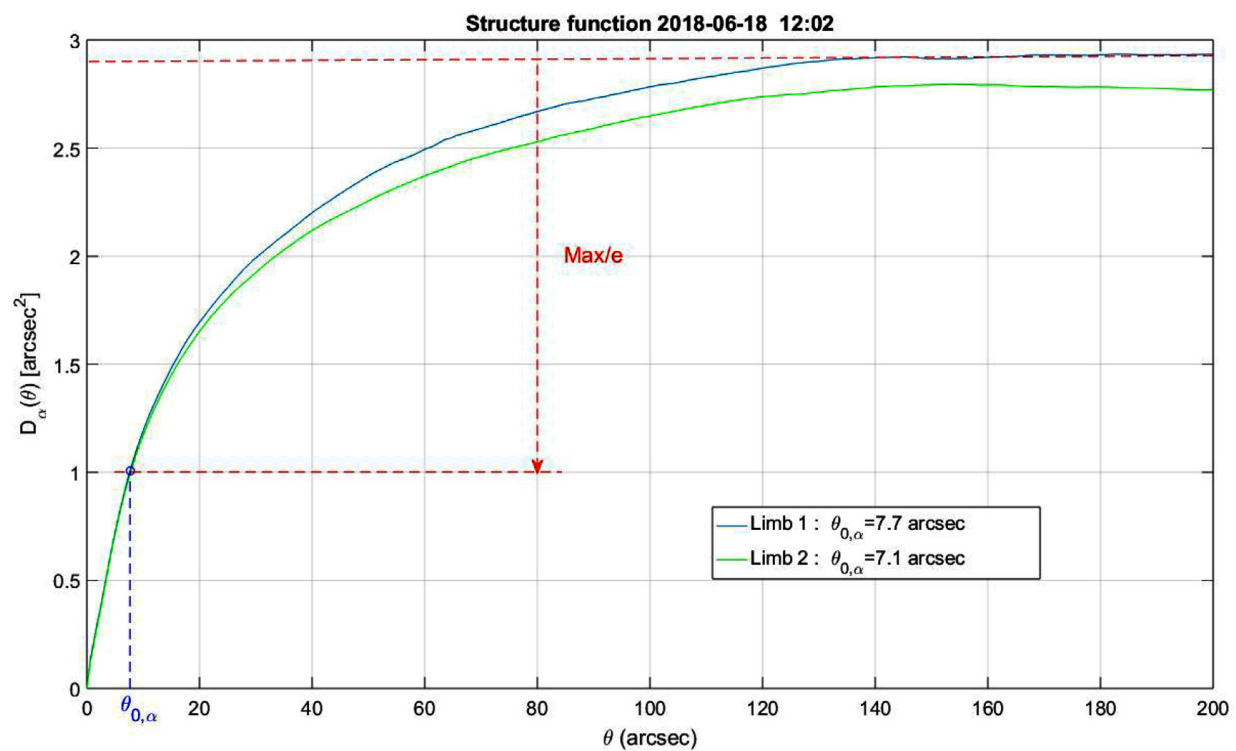

Figure 1. Example of AA fluctuation structure function obtained with the PML instrument on the Sun edge at the Calern Observatory on 2018 June 18.
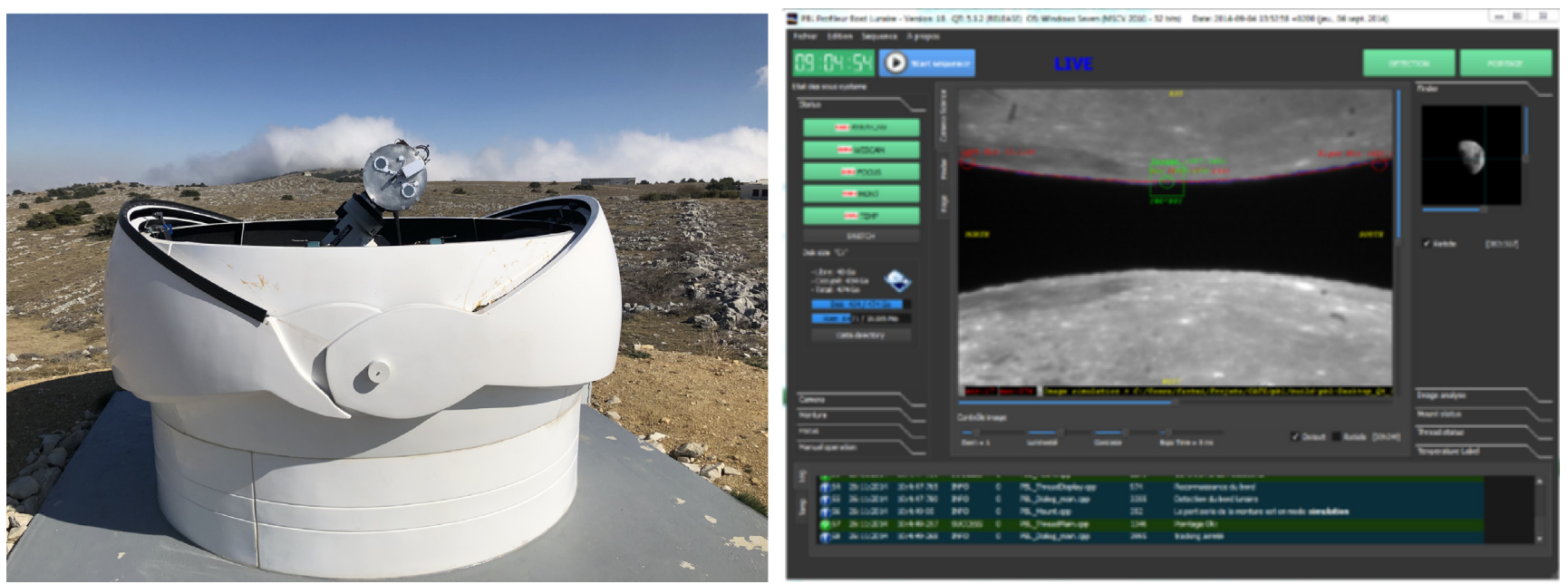

Figure 2. Left: the PML instrument inside its $12 \mathrm{ft}$ AllSky dome. Right: the graphical user interface of the PML instrument showing the two Moon limb images.

For the error on the $\theta_{0}$ estimation, one can use the same reasoning than for the coherence time in Aristidi et al. (2019). The error on $\theta_{\alpha}$ deduced from the structure function $D_{\alpha}(\theta)$ is related to the finite difference around $\theta=\theta_{\alpha}$ as

$\Delta \theta_{\alpha} \simeq \frac{\Delta D_{\alpha}}{D_{\alpha}^{\prime}\left(\theta_{\alpha}\right)}$

where $D_{\alpha}^{\prime}$ is the first derivative of the structure function $D_{\alpha}$. The error $\Delta D_{\alpha}$ on $D_{\alpha}$ is obtained as the standard deviation of the structure function in the saturation zone (typical values are of 1.5 per cent), which is low as seen in Fig. 1 . The derivative $D_{\alpha}^{\prime}\left(\theta_{\alpha}\right)$ is deduced from the slope of the structure function around $\theta_{\alpha}$. Errors $\Delta \theta_{\alpha}$ were computed for each of the two limb's (Fig. 2) structure functions, on more than 3000 acquisitions for the period of 2018 July-December leading to a typical error of $\sim 6.4$ per cent.

The error on $\theta_{\alpha}$ leads to an error $\Delta \bar{h}_{\alpha}$ on the equivalent altitude (equation 3) obtained by differentiation of equation (11) or (12). For an outer scale considered constant, the relative error on the equivalent altitude is similar to the one on $\theta_{\alpha}$ where a typical value as said above is of $\sim 6.4$ per cent. In addition, the equivalent altitude $\bar{h}$ deduced from equations (11) and (12) needs an estimate of the outer scale $\mathcal{L}_{0}$. However, as the outer scale is not yet available from the PML instrument, we use for the moment a standard value $\mathcal{L}_{0}=20 \mathrm{~m}$. This induces a bias $\Delta \bar{h}_{L_{0}}$ on the equivalent altitude, which is of 7.0 per cent for outer scales $\mathcal{L}_{0}$ varying from 10 to $40 \mathrm{~m}$ covering the major situations on traditional sites (Ziad et al. 2000). Combining these two contributions, the relative uncertainty on $\bar{h}$ is then $\frac{\Delta \bar{h}}{\bar{h}} \simeq 13.4$ per cent.

Finally, the error $\Delta \theta_{0}$ on the isoplanatic angle $\theta_{0}$ is obtained by introducing the above $\bar{h}$ uncertainty in equation (4). Knowing that $r_{0}$ is obtained in the PML instrument from a DIMM method (Ziad et al. 2013) where usually the prevailing error is statistical for Differential Image Motion Monitor (DIMM) monitors (Aristidi et al. 2019) but for the PML more than $600 \times 1000$ samples are used leading to a statistical error lower than 0.2 per cent. Thus, added to the other contributions on the error on $r_{0}$ estimation, the uncertainty 

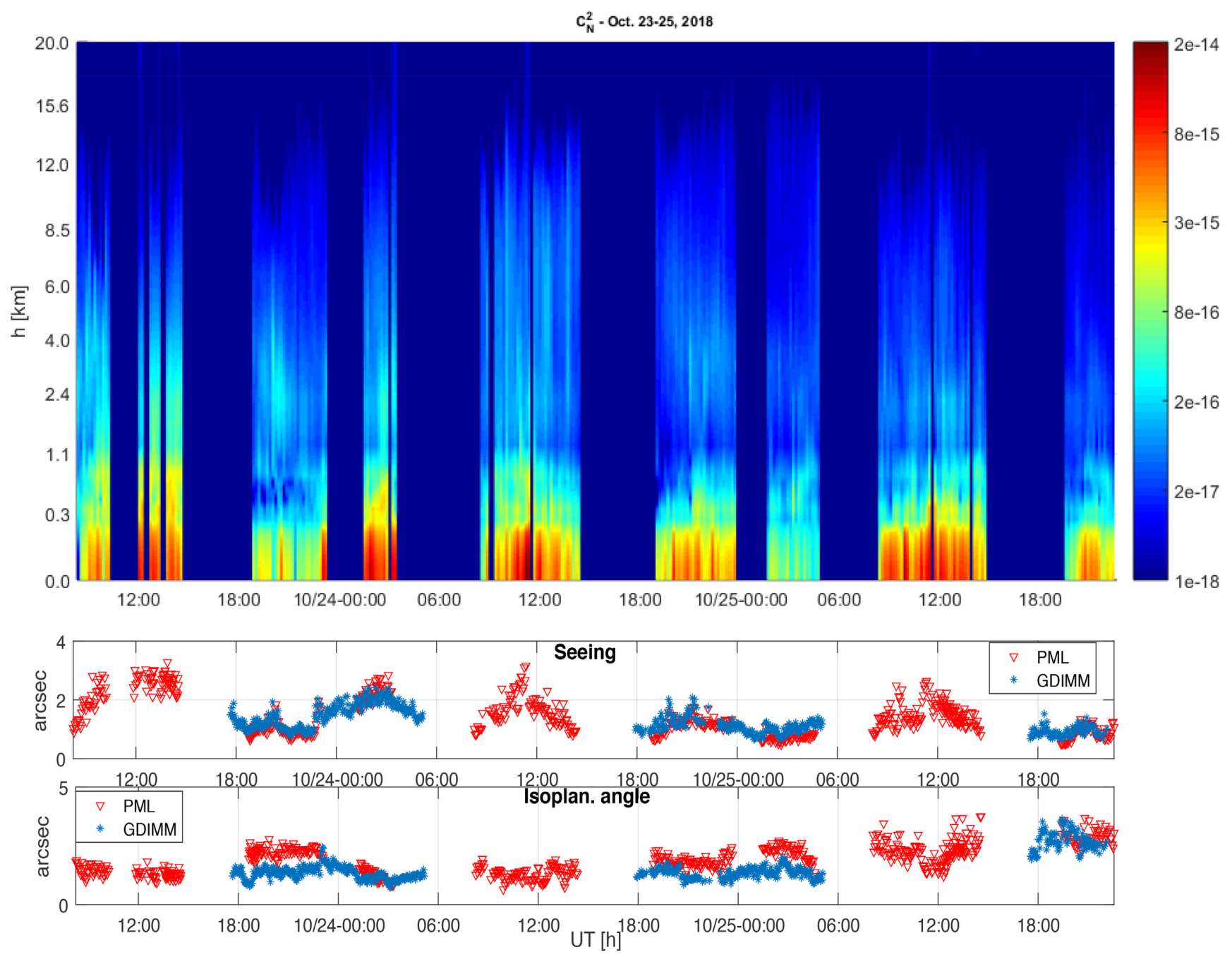

Figure 3. Turbulence $C_{n}^{2}$ profiles for the period of 2018 October $23-25$ obtained with the PML instrument at the Calern Observatory (Top). The altitude is given above the Calern Observatory $(1270 \mathrm{~m})$. Observations have been obtained on the Sun edge between $7 \mathrm{am}$ and $5 \mathrm{pm}$ and on the Moon limb for the rest of the time. Bottom: Corresponding seeing and isoplanatic data obtained from the PML $C_{n}^{2}$ profiles above and compared with the GDIMM measurements. The isoplanatic PML results are obtained using the $C_{n}^{2}$ profiles when GDIMM $\theta_{0}$ is deduced from scintillation.

on Fried parameter is of $\sim 1$ per cent. Then, the total error on the isoplanatic angle estimation from this new PML technique is of $\simeq 14.4$ per cent.

\section{INSTRUMENTS OF ISOPLANATIC DOMAIN ESTIMATION}

\subsection{PML instrument}

The PML instrument is dedicated to the extraction of the $C_{n}^{2}$ profile with high vertical resolution from lunar (or solar) limb fluctuations. The PML instrument is based on a differential method by observation of the lunar (or solar) limb through two subapertures (Fig. 2). The Moon or Sun limb acts as a continuum of double stars with all possible angular separations required between two points to scan the atmosphere with a very fine resolution.

The PML instrument consists of a 16 inch telescope (Meade M16) installed on an Astrophysics AP3600 mount. The pupil mask, composed of two subapertures of diameter $D=6 \mathrm{~cm}$ separated by a baseline $B=26.7 \mathrm{~cm}$, is placed at the entrance pupil of the telescope (Fig. 2). An optical device is installed in the focal plan of the telescope (Ziad et al. 2013; Catala et al. 2017; Chabé et al. 2019) consisting mainly of a Dove prism to reverse one of the two images of the lunar (solar) edge avoiding an overlapping of the two images of the Moon/Sun (Fig. 2). The two images of the Moon limb (or Sun edge) are acquired with a CCD camera (Fig. 2), the exposure time is set to $5 \mathrm{~ms}$. The AA fluctuations are measured perpendicularly to the lunar (or solar) limb. Then, a transverse covariance $C_{\Delta \alpha}(\theta)$ of the difference of the AA fluctuations $\alpha$ between the two images of the lunar (or solar) limb (Fig. 2) is deduced. After developments, this angular covariance is given by (Ziad et al. 2013)

$C_{\Delta \alpha}(\theta)=\int \mathrm{d} h C_{n}^{2}(h) K_{\alpha}(B, h, \theta)$,

where the kernel $K_{\alpha}(B, h, \theta)=2 C_{\alpha}(\theta h)-C_{\alpha}(B-\theta h)-C_{\alpha}(B+$ $\theta h)$ is a triplet of normalized spatial covariances, which in the case of the von Kàrmàn model for a baseline $\varrho$, a subaperture diameter $D$ (here $6 \mathrm{~cm}$ ), and a single layer at altitude $h$ is given in equation (9).

The spatial covariance triplet above is similar to the Scidar's one (Fuchs, Tallon \& Vernin 1998). The position of the lateral peaks defines the altitude of the turbulent layer $(B= \pm \theta h)$ so that its 
height leads to the contribution of this layer to the $C_{n}^{2}(h)$ profile. For the whole atmosphere, we have a superposition of different triplets corresponding to different turbulent layers (see fig. 6 of Ziad et al. 2013, as an example of illustration).

The $C_{n}^{2}(h)$ profiles are retrieved by solving an inverse problem (Blary 2015; Ziad et al. 2018). The minimization process makes use of a regularization constraint based on the gradient. A full description of the algorithm will be published in a forthcoming paper (Chabé et al. 2019).

The PML acquisition software provides a real-time computation of the $C_{n}^{2}$ profile with a high-resolution vertical every $2 \mathrm{~min}$. In addition, the PML instrument is now equipped with automatic panels to cover the two subapertures with solar filters (Fig. 2) for a fast and automatic switch from night/Moon observation to day/Sun observation (Ziad et al. 2018). We then have a unique instrument to study the turbulence conditions during the daytime/night-time transition as shown in Fig. 3. Other parameters of turbulence are also accessible from this instrument such as the profile of outer scale, the seeing, and the isoplanatic angle. For the latter, two techniques described in Section 2 are used. The first and easiest one is based on the $C_{n}^{2}$ profile to deduce first the $\bar{h}$ from equation (3), which is introduced into equation (2) to provide an estimation of $\theta_{0}$. The second method is based on the angular structure functions of AA fluctuations $D_{\alpha}(\theta)$ deduced from Moon (or Sun) limb as shown in Fig. 1. The saturation level is obtained directly from $D_{\alpha}(\theta)$ for large angular separations $\theta$ as shown in Fig. 1. The fold of $D_{\alpha}(\theta)$ by a constant $\kappa=e$ leads to $\theta_{\alpha}$. Including this value of $\theta_{\alpha}$ in equations (11) or (12), the equivalent altitude $\bar{h}$ is obtained. Then, equation (2) gives the isoplanatic angle $\theta_{0}$ estimation.

\subsection{Scintillation-based instruments}

One of the easiest and low-cost way to estimate the isoplanatic angle is the use of scintillation of single star through a small telescope (Section 2). Thus, different instruments based on scintillation measurement for the estimation of $\theta_{0}$ have been developed during the last $20 \mathrm{yr}$. Among such instruments, there is the GSM (Generalized Seeing Monitor; Ziad et al. 2000) and recently the GDIMM (Generalized Differential Image Motion Monitor), a compact instrument aiming at replacing the aging GSM (Aristidi et al. 2014; Ziad et al. 2018; Aristidi et al. 2019). These instruments lead to estimates of the seeing $\epsilon_{0}$, outer scale $\mathcal{L}_{0}$, and coherence time $\tau_{0}$ from statistics of AA fluctuations, while scintillation with a special pupil filtering scheme provides isoplanatic angle $\theta_{0}$ estimations. Indeed, these instruments measure the scintillation of a bright star using an aperture of $10 \mathrm{~cm}$ with a central obstruction of $4 \mathrm{~cm}$. Then, this scintillation index is introduced in equation (6) and knowing the zenithal angle, estimates of $\theta_{0}$ are deduced. A correction for finite exposure time on scintillation measurements is performed; it consists in computing scintillation statistical moments for 5 and $10 \mathrm{~ms}$ and in extrapolating linearly to the $0 \mathrm{~ms}$ exposure time (Ziad et al. 2000; Aristidi et al. 2014).

Since 1997 and for more than $15 \mathrm{yr}$, GSM was used in a large number of astronomical observatories and for prospecting potential new sites (Ziad et al. 2000). GDIMM is a part of the Calern Atmospheric Turbulence Station (CATS; Chabé et al. 2016; Ziad et al. 2018; Aristidi et al. 2019), which was developed as a site-monitoring facility at the Plateau de Calern (France). The instrument is running routinely since the end of 2015 and provides a measurement of turbulence parameters every $2 \mathrm{~min}$.

\section{ISOPLANATIC DATA AND COMPARISONS}

In this section, we present results of isoplanatic angles obtained with the PML instrument by means of a new technique; results of $\theta_{0}$ obtained with the technique based on angular structure function (Section 2) lead to an estimation of $\theta_{0}$, which we compare to isoplanatic angles obtained from the scintillation techniques used on GSM and GDIMM instruments and to estimations of the isoplanatic angle from $C_{n}^{2}$ profiles from the PML data. Simultaneous measurements of $\theta_{0}$ from both PML techniques and GSM/GDIMM scintillation method are discussed.

Fig. 3 shows an example of the turbulence profile evolution with the PML throughout the daytime and the night-time for the period of 2018 October 23-25 at the Calern Observatory. The figure shows the full PML profiles with 33 layers from the Sun between 7 am and $5 \mathrm{pm}$ and the rest of the time from the Moon. The resolution obtained by the PML is $\Delta h=100 \mathrm{~m}$ for the ground layer ( $h \leq$ $1 \mathrm{~km}), \Delta h=500 \mathrm{~m}$ for the low free atmosphere $(1 \mathrm{~km}<h<5 \mathrm{~km})$, $\Delta h=1000 \mathrm{~m}$ for the mid-free atmosphere $(5 \mathrm{~km}<h<15 \mathrm{~km})$, and $\Delta h=2000 \mathrm{~m}$ for the high free atmosphere $(h>15 \mathrm{~km})$. The highest altitude $h_{\max }$ reachable with the PML is more than $50 \mathrm{~km}$ but we limited $h_{\max }$ to $25 \mathrm{~km}$ since the turbulence is very low above this altitude. The limited field of view of the PML instrument limits the lowest measurable altitude to around $100 \mathrm{~m}$. The contribution of the lowest layer $0-100 \mathrm{~m}$ is obtained by the difference between the profile deduced from the inversion of the PML covariances and the total seeing from DIMM method (Section 2) using PML data. For the total seeing obtained from PML, we have about 620 estimations (each point of the Moon limb leads to a DIMM measurement) and we keep only the median one (middle panel of Fig. 3). This PML seeing measurement is in excellent agreement with the GDIMM's data as shown in the middle panel of Fig. 3. Furthermore, the $C_{n}^{2}$ profiles and Fried parameters obtained with PML are used to provide the isoplanatic angle estimations as explained in Section 2 by using equations (3) and (2). These $\theta_{0}$ estimations from PML instrument are presented in the bottom panel of Fig. 3. It is worth noting that isoplanatic values improve at night with respect to daytime, because the seeing conditions progressively improve when switching from the Sun to the Moon observations. But on average, daytime and night-time $\theta_{0}$ are comparable since it is dominated by the high layers in the atmosphere, which are similar for daytime and night-time because they only depend on large-scale meteorological phenomena. It is also interesting to point out a correlation between seeing and isoplanatism, particularly around noon, which implies that the high altitude turbulence contribution to the seeing is not negligible compared to the ground layer: Around noon is when the worst seeing conditions occur with turbulence occurring up to $20 \mathrm{~km}$, as shown on the $C_{n}^{2}$ profiles in Fig. 3 .

We note that the isoplanatic results deduced from scintillation (GDIMM data in Fig. 3) show some discrepancies with those obtained from PML $C_{n}^{2}$ profile. For instance, the mean value of the relative difference between GDIMM and PML in terms of isoplanatic angle is of 46.1 per cent, but the standard deviation is 30.1 per cent for the whole period of 2018 October 23-25 (Fig. 3). Such a difference is significant and needs to be addressed before being able to draw conclusions regarding the different techniques. Two issues have been raised; first, the GDIMM technique is based on scintillation measurement very sensitive to clouds that are sometimes not very visible such as the high-altitude cirrus. The second point concerns the impact of the Moon on the scintillation measurements, knowing that the comparison between PML and GDIMM is only possible when the Moon is observable. To figure 

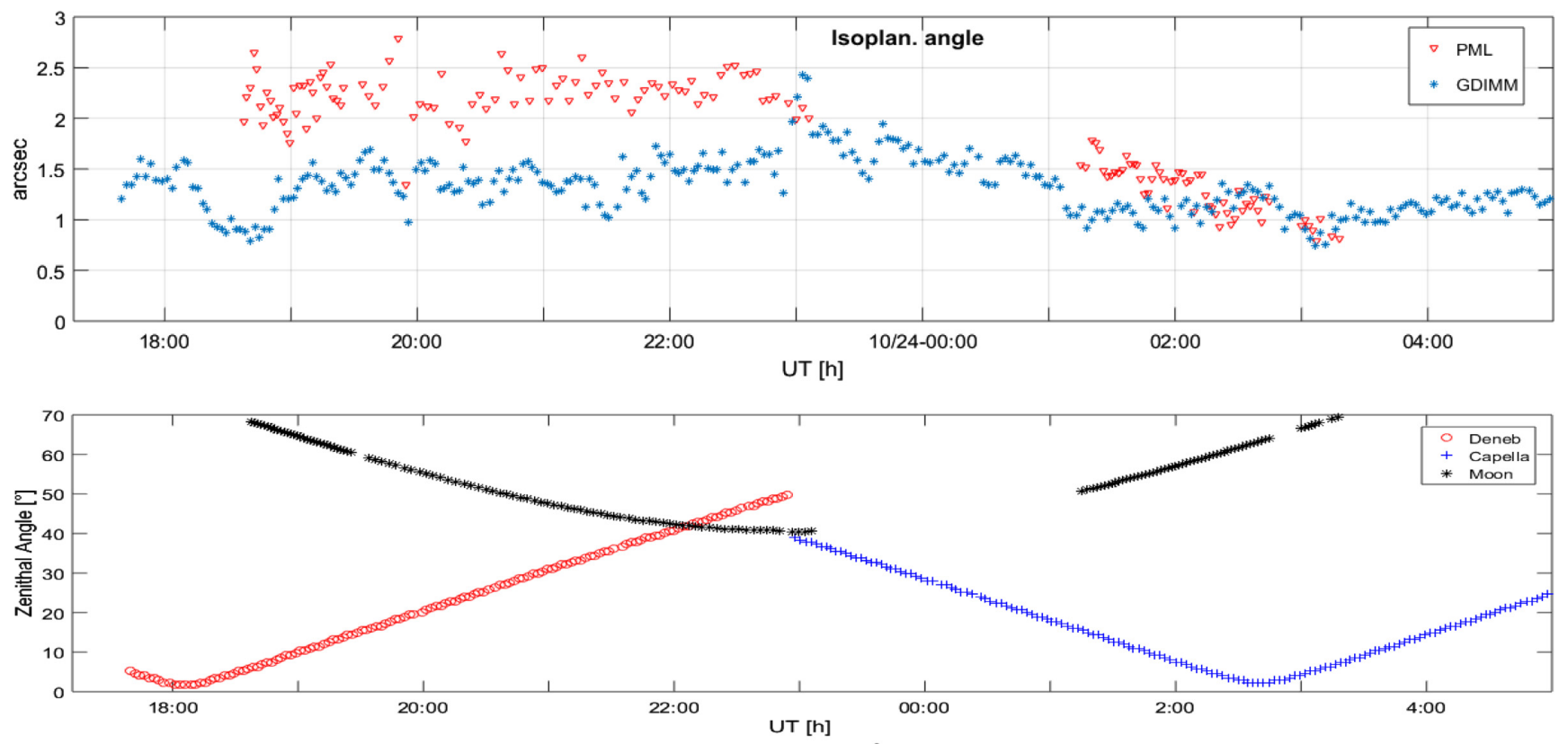

Figure 4. Top: comparison of isoplanatic angle deduced from PML $C_{n}^{2}$ profiles and GDIMM scintillation measurements for the night of 2018 October 23. Bottom: zenithal angle of the Moon during the PML observations and of the stars observed by the GDIMM instrument.

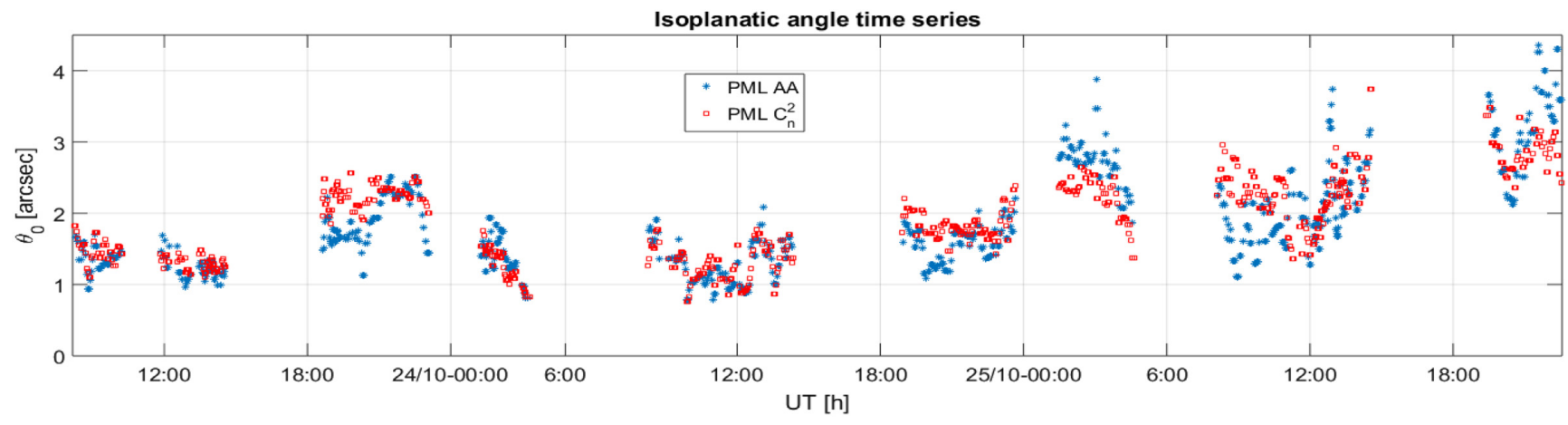

Figure 5. Comparison of isoplanatic results obtained from the PML techniques at the Calern Observatory. The PML $\theta_{0}$ are obtained using the $C_{n}^{2}$ profiles (red) and AA structure function (blue).

out this issue and for more clarity, let us focus on the night of October 23 as presented in Fig. 4. In addition to the isoplanatic angle estimations from PML and GDIMM instruments, we plotted the zenithal angles of the Moon and the observed stars with the GDIMM instrument. The first remark is the correlation between the isoplanatic angle obtained from scintillation measured with the GDIMM and the zenithal angle of the Moon. This correlation is the result of the conjunction of the positions in the sky of the Moon and the observed star with the GDIMM. In the beginning of the night, the Moon was rising and the GDIMM was observing Deneb ( $\alpha$ Cygni) before its meridian transit. Thus, the Moon illuminated directly the pupil of the GDIMM, which induced fluctuations in the intensity of the spots on the CCD camera. These intensity fluctuations are added to those induced by the atmosphere and leading to a reduced $\theta_{0}$ as shown in the first part of the night on the top panel of Fig. 4. GDIMM changed the star at 10:58 pm, passing from Deneb to Capella ( $\alpha$ Aurigae) that was at $39^{\circ}$ from the zenith in the opposite direction (north-east) to the Moon that was just transiting. In this configuration, the Moon was not directly illuminating the pupil of the GDIMM and this explains why the GDIMM $\theta_{0}$ immediately started matching the PML values. The observed downward trend of $\theta_{0}$ is simply a normal degradation of turbulence conditions in the upper layers because this phenomenon is also observed on PML values and coincides with increasing seeing (Fig. 3). The differences between the two instruments around 1:30 pm remain within the error bars (Aristidi et al. 2019). When the Moon was setting, the GDIMM and PML $\theta_{0}$ were in excellent agreement. The GDIMM targets and their chronology are not transposable from one night to the next, so on October 24, the order in which GDIMM targets were observed was Vega ( $\alpha$ Lyrae), Deneb, and Capella when the Moon rose more than 30 min later. To prevent the PML telescope from striking the pillar, the mount changes the position of the telescope from west to east. After this operation, the mount had difficulties pointing the Moon due a balancing problem, which explains why data are missing in the middle part of the night. This problem has been since resolved.

The new and direct technique that we developed for PML is based on the reconstruction of the angular AA structure function (Section 2). Fig. 5 shows the results of $\theta_{0}$ obtained from AA structure functions and those deduced from the $C_{n}^{2}$ profiles for the 

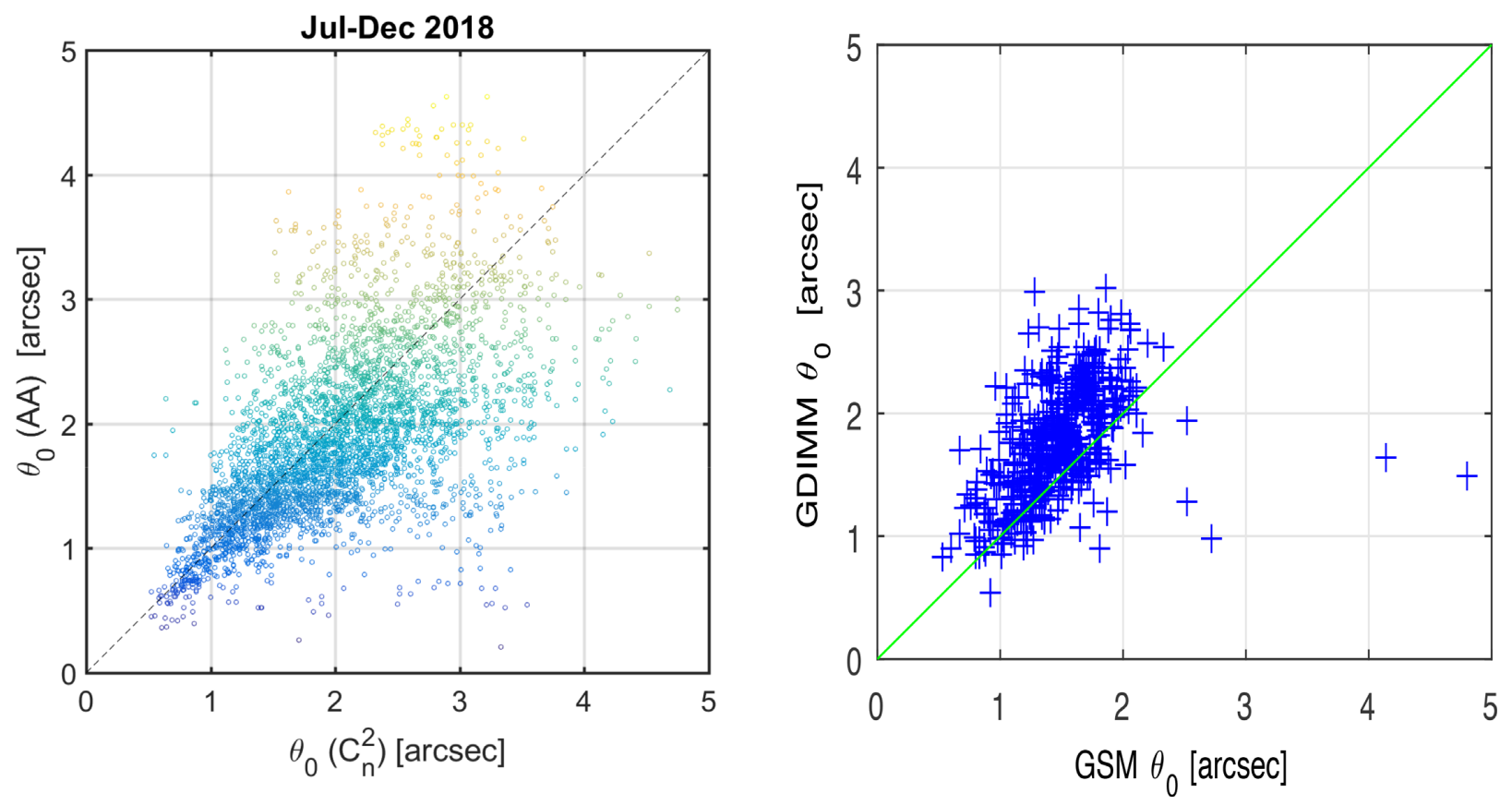

Figure 6. Comparison of isoplanatic results obtained from different instruments at the Calern Observatory. Left-hand panel: comparison of PML data using AA structure function method and $C_{n}^{2}$ profiles for the period of 2018 July-December. 4414 data are represented, consisting of 1364 measurements on Moon limb and 3050 on Sun edge observations. Right-hand panel: comparison of $\theta_{0}$ results obtained from the GSM and GDIMM measurements in 2015. Both the instruments use scintillation measurement for the extraction of $\theta_{0}$.

same period than Fig. 3, i.e. from 2018 October 23 to 25. We point out that $\theta_{0}$ estimations from this new technique based on the AA structure function are in good agreement with those obtained from the $C_{n}^{2}$ profiles. However, some differences are visible particularly in the night of October 25, for example around 3 am and after $10 \mathrm{pm}$. In addition, one can see that $\theta_{0}$ obtained from AA structure functions are dispersed compared to those deduced from $C_{n}^{2}$ profiles and this happens when the seeing conditions are good (see the middle panel of Fig. 3). The lower the seeing, the smaller the AA fluctuations, which means that the effect of instrumental noise becomes more important in the reconstruction of the structure function. Although also obtained from the same AA fluctuations, the $C_{n}^{2}$ profiles and seeing are less affected because they use differential techniques (Section 3). In general, $\theta_{0}$ obtained from both techniques using the PML instrument are coherent when using more statistics as shown in Fig. 6 (left-hand panel). These data have been obtained at the Calern Observatory for the period of 2018 July-December, consisting of 4414 data distributed between Moon limb and Sun edge observations. One can note that there is a good agreement for small $\theta_{0}$ values and progressively the discrepancy is larger when the isoplanatic angle tends to large values. Good turbulence conditions (large $\theta_{0}$ ) reduce AA fluctuations making their detection with the PML less precise, particularly for the AA structure function reconstruction.

Finally, we would like to point out in this paragraph that even when we use the same technique but with different equipment and/or data processing, the results are not identical. In 2015, simultaneous data sets of isoplanatic angle were obtained at the Calern Observatory from two the instruments, GDIMM and GSM, installed on the same platform and separated by a distance lower than $1 \mathrm{~m}$. Even if these instruments use the same technique to extract $\theta_{0}$, the scintillation is not measured exactly in the same way in both the instruments. The main difference of scintillation measurement is due to the intensity technique and the used detector. Indeed, the GSM uses an interferometric method with a photomultiplier (Ziad et al. 2000) whereas the GDIMM measures the intensity of the observed star image in the telescope focal plane with a CCD. The latter has a non-homogeneous sensitivity of the pixels that are less sensitive than a photomultiplier; there is a residual drift due to the diurnal movement, which implies a drift of the images on the CCD pixels. Thus, due to its interferometric technique and its highsensitivity detector, the GSM provides a more precise scintillation measurement and then isoplanatic angle estimation. In addition, the exposure time effect debiasing is more pertinent with the GSM than with the GDIMM. Indeed, in the GSM, $10 \mathrm{~ms}$ exposure intensity is obtained by binning two contiguous $5 \mathrm{~ms}$ exposures. However, the GDIMM starts acquiring a sequence of $N$ images with a $5 \mathrm{~ms}$ exposure and then immediately another sequence with a $10 \mathrm{~ms}$ exposure is recorded. Indeed, comparisons between GSM and GDIMM show in general (right-hand panel of Fig. 6) that GDIMM provides a slight overestimate of isoplanatism, compared to GSM, due to all the reasons above.

\section{CONCLUSIONS}

The first direct technique for the estimation of the isoplanatic angle has been presented. This technique is based on the angular correlation of AA fluctuations along an extended object such as the Moon or the Sun. This technique provides continuous and consistent results, allowing us to measure $\theta_{0}$ continuously during the transition between daytime and night-time conditions for the first time, thanks to the Sun and Moon edges. Comparisons with existing techniques using different observable quantities and instruments have been performed. These comparisons allowed us to show the impact of the Moon on the isoplanatic angle estimated from scintillation 
measurements. Indeed, this impact could lead to a relative difference of 46.1 per cent between GDIMM and PML in terms of isoplanatic angle. It has been also shown that this new PML technique could show unsteady results when the seeing conditions are excellent due to reduced AA fluctuations. But this problem can be overcome by enhancing the PML sensitivity. Different solutions are possible such as extending the focal distance with a Barlow lens although this reduces the field and increases the lowest detected altitude. Another option is the use of a larger camera. A new Basler camera, cheaper but with twice field size, is currently under testing to replace the existing one. Instruments using same scintillation technique such as GSM and GDIMM provide coherent estimations of $\theta_{0}$ but not identical results depending on the detector sensitivity and exposure time correction.

\section{ACKNOWLEDGEMENTS}

We express our gratitude to Dr. Olivier Lai for his valuable help on the review of english text and effective exchanges. We would like to thank the MéO team and Calern technical staff. The CATS project has been done under the financial support of CNES, Observatoire de la Côte d'Azur, Labex First TF, AS-GRAM, Federation Doëblin, Université de Nice-Sophia Antipolis, and Région Provence Alpes Côte d'Azur.

\section{REFERENCES}

Aristidi E., Fanteï-Caujolle Y., Ziad A., Dimur C., Chabé J., Roland B., 2014, in Larry M. S., Roberto G., Helen J. H., eds, Proc. SPIE Conf. Ser. Vol.
9145, Ground-based and Airborne Telescopes V. SPIE, Bellingham, p. 91453G

Aristidi E., Ziad A., Chabé J., Fantëi-Caujolle Y., Renaud C., Giordano C., 2019, MNRAS, 486, 915

Avila R., Ziad A., Borgnino J., Martin F., Agabi A., Tokovinin A., 1997, J. Opt. Soc. Am., 14, 3070

Blary F., 2015, Caractérisation et Modélisation de la Turbulence Optique dans un espace confiné, PhD thesis, Université de Nice Sophia Antipolis Catala L. et al., 2017, MNRAS, 467, 3699

Chabé J., Ziad A., Fanteï-Caujolle Y., Aristidi E., Renaud C., Blary F., Marjani M., 2016, in Helen J. H., Roberto G. Heather K. M., eds, Proc. SPIE Conf. Ser. Vol. 9906, Ground-based and Airborne Telescopes VI. SPIE, Bellingham, p. $99064 \mathrm{Z}$

Chabé J., Aristidi E., Ziad A., Lantéri H., Fanteï-Caujolle Y., Giordano C., Borgnino J., Renaud C., 2019, submitted to Applied Optics

Fried D. L., 1979, Opt. Acta, 26, 597

Fuchs A., Tallon M., Vernin J., 1998, PASP, 110, 86

Loos G., Hogge C., 1979, Appl. Opt., 18, 2654

Roddier F., Gilli J. M., Vernin J., 1982, J. Opt., 13, 63

Ziad A. et al., 2013, A\&A, 559, L6

Ziad A., Conan R., Tokovinin A., Martin F., Borgnino J., 2000, Appl. Opt., 39,5415

Ziad A., Borgnino J., Dali Ali W., Berdja A., Maire J., Martin F., 2012, J. Opt., 14, 45705

Ziad A., Chabé J., Fanteï-Caujolle Y., Aristidi E., Renaud C., Ben Rahhal M., 2018, in Laird M. C., Laura S., Dirk S., eds, Proc. SPIE Conf. Ser. Vol. 10703, Adaptive Optics Systems VI. SPIE, Bellingham, p. $107036 \mathrm{~L}$

This paper has been typeset from a $\mathrm{T}_{\mathrm{E}} \mathrm{X} / \mathrm{L} \mathrm{T} \mathrm{E} \mathrm{X}$ file prepared by the author. 